\title{
Incidence and Prognosis of Bone Metastasis in Newly Diagnosed Differentiated Thyroid Carcinoma: A SEER Population-Based Study
}

\section{Lin Qi}

Second Xiangya Hospital of Central South University

\section{Wenchao Zhang}

Second Xiangya Hospital of Central South University

\section{Xiaolei Ren}

Second Xiangya Hospital of Central South University

Chaoqian Liu

Second Military Medical University

\section{Chao Tu}

Second Xiangya Hospital of Central South University

Zhihong Li ( $\square$ lizhihong@csu.edu.cn )

Second Xiangya Hospital of Central South University

\section{Research Article}

Keywords: bone metastasis, differentiated thyroid carcinoma, incidence, prognosis, SEER

Posted Date: February 23rd, 2021

DOl: https://doi.org/10.21203/rs.3.rs-191160/v1

License: (1) This work is licensed under a Creative Commons Attribution 4.0 International License. Read Full License 


\section{Abstract}

Background: Clinical characteristics and sociodemographic of differentiated thyroid carcinoma (DTC) patients with bone metastasis (BM) remain unclear.

Methods: Data of patients with newly diagnosed DTC from 2010 to 2016 were retrieved from Surveillance, Epidemiology, and End Results (SEER) database. Incidence trend of BM in patients with DTC was explored by joinpoint regression. Multivariable logistic regression was utilized to figure out predictors of BM at initial presentation of DTC, and was further evaluated by receiver operator characteristics (ROC) analysis. Multivariable Cox regression was applied to reason out factors associated with overall survival and DTC-specific survival.

Results: A total of 67,176 patients with DTC were identified from the database, with $0.36 \%(244 / 67176)$ presenting BM at the time of diagnosis. The age-adjusted incidence of BM in DTC was relatively stable during study period with an average annual percentage change (AAPC) of 2.52. Multivariable logistic regression recognized eight factors as predictors of presence of $\mathrm{BM}$ among the entire cohort.

Multivariable Cox regression indicated multiple metastatic sites, total thyroidectomy and radioisotopes as predictors for survival of patients.

Conclusions: The incidence of BM in DTC patients has been stable in recent years. Predictors and prognostic factors of BM in DTC patients were identified in this study.

\section{Background}

The overall incidence of thyroid carcinoma has been on the rise in recent decades[1], and it has been reported that the newly diagnosed thyroid carcinoma cases in 2018 was 567,233 worldwide[2]. Furthermore, new cases of thyroid carcinoma in 2020 will reach up to 52,890 in United States according to the newly published cancer statistics[3]. DTC is the most common type of thyroid cancer, as well as the most curable endocrine tumors with 10-year cancer-specific survival of more than 70\%[4]. Distant metastasis in DTC was extremely rare and has been regarded as predictor for poor prognosis[5]. It has been demonstrated that 10-year overall survival rate of DTC patient with distant metastasis was less than $50 \%$, lower than that of no metastasis[6].

Second to lung, bone was reported to be one of the most common sites for metastasis of thyroid carcinoma, and it was still of great challenge to improve the prognosis in DTC patients with BM [7]. Spine (34.6\%), pelvis (25.5\%), sternum and ribs (18.3\%), extremities $(10.2 \%)$, shoulder girdle $(5.4 \%)$, and craniomaxillofacial (5.4\%) were the most common sites of BM in DTC[8]. Although BM was associated with poor prognosis, early detection and administration of appropriate therapy could significantly alleviate suffering of patients[9]. Treatments including radiotherapy, chemotherapy, and surgery have been applied or reported to improve outcomes of DTC patients with BM[9-11]. For instance, chemotherapy drugs such as Zoledronic Acid has been demonstrated to be effective in reducing skeletal- 
related events or delaying its appearance in patients with BM from DTC[7]. Moreover, bone stability of patients could be improved by using percutaneous osteoplasty combined with radioiodine therapy[12].

Early detection or prediction of BM in DTC was in urgent need for improving the prognosis as well as reducing unnecessary cost of patients. With early intervention, BM can be controlled or suppressed. Nevertheless, the promising data related to the incidence and prognosis of BM in newly diagnosed DTC, especially the population-based study, was extremely limited[9, 13, 14]. Even though characteristics of BM from DTC have been discussed in some case reports or reviews, we cannot reconcile these controversial observations to make a conclusion[15-17]. Thus, we extracted data from the SEER database from 20102016 to conduct this population-based analysis, aiming to identify the trend in incidence and investigate potential predictors and prognostic factors of BM in newly diagnosed DTC patients.

\section{Methods}

\section{Data source}

SEER database was one of the most authoritative cancer databases worldwide, which collected individual data of cancer patients from various regional cancer registries throughout the United States since 1973, covering approximately 30 percent of the U.S. population[18]. Data in SEER database was deemed reliable ascribe to rigorous quality control studies and various data assessments. We obtained data of information of eligible patients in this study by using the SEER ${ }^{\star S t a t}$ software version 8.3.6 (Surveillance Research Program, National Cancer Institute, seer.cancer.gov/seerstat). The SEER database provided data of patients up to 2016 based on the Nov 2018 submission, and data on specific metastatic sites including lung, bone, liver and brain was available since 2010. Therefore, the study period was set between Jan 2010 to Dec 2016.

\section{Study design and participants}

Patients with BM of newly diagnosed DTC during 2010 to 2016 were initially enrolled in this study and then screened by following the inclusion criteria below. (1) Age more than18 years old, (2) Diagnosis of DTC with positive histology conformation (ICD-0-3 histology code: 8050, 8052, 8260, 8330-8335 and 8340-8344) (3) DTC as the only primary cancer, (4) known survival status and time. Data of individual patient were excluded if there was no specific information of TNM system and bone or lung metastasis. In this study, variables collected through SEER database were composed of sociodemographic characteristics (year at diagnosis, age at diagnosis, race, gender, insurance situation and marital status), clinicopathological characteristics (laterality, histologic, American joint committee on cancer (AJCC) clinical stage, AJCC TNM classification, extraskeletal metastasis) and treatment-related information (surgical procedures, radiotherapy and chemotherapy). All data was confirmed by two independent authors (Lin Qi and Wenchao Zhang). Age was divided into 4 intervals ( $\leq 44,45-54,55-64$ and $\geq 65$ ) based on the major age of onset. Race was classified into white, black and others. Year of diagnosis was stratified into two period (2010-2013 and 2014-2016). The primary endpoint in this study were overall 
survival and cancer-specific survival, defined as time from onset to death caused by all reasons and cancer respectively. The study design with corresponding process was shown in Fig. 1.

\section{Statistical analysis}

Description analysis was utilized to present the overall view of frequency and age-adjusted incidence of BM in newly diagnosed DTC. Frequency was defined as the percentage of BM among the entire cohort or in the cases with distant metastasis. We principally focused on the entire cohort rather than the subset with metastatic disease because this was a population-based study. Sociodemographic, clinicopathological and treatment-related characteristics including year at diagnosis, age at diagnosis, race, gender, insurance situation, marital status, laterality, histologic, AJCC clinical stage, AJCC TMN classification, multifocality, extra skeletal metastases, surgery, radiation therapy and chemotherapy were employed to stratify the data. Survival data was expressed in the median with interquartile range. Incidence of DTC patients with BM were age adjusted to the 2000 US standard population and expressed per 1000000 person-years. Joinpoint Regression Analysis Program (version 4.7.0.0; National Cancer Institute) was used to calculate annual percentage change (APC) and corresponding $95 \%$ Cls to present the trend in incidence.

The differences in sociodemographic, clinicopathological and treatment characteristics were compared by Pearson's chi-squared test. Univariable and Multivariable logistic regression were applied to figure out possible predictors of BM and build the predicting models. Corresponding Odds ratio (OR) along with $95 \%$ confidence intervals $(\mathrm{Cl})$ were also presented. The models were further assessed by using the ROC curves. Meanwhile, the nomogram was established based on the significant predictors to predict the probability of BM in DTC patients. Furthermore, univariable and multivariable cox regression were conducted to identify prognostic factors for overall survival and cancer-specific survival in DTC patients with BM. Kaplan-Meier curves along with log-rank tests were created according to the significant prognostic factors. All statistical analyses were done among both the entire cohort and subset with metastatic disease and were conducted utilizing SPSS version 24 for Windows (IBM, Armonk, New York, USA). Survival curves were generated by GraphPad Prism 8 . The nomogram was established using $\mathrm{R}$ version 3.5.3. Two-sided $P<0.05$ was defined as statistical significance.

\section{Results}

\section{Characteristics of enrolled patients}

A total of 67,176 patients with DTC in United states during 2010 to 2016 were screened from SEER database based on the inclusion criteria. Among these patients, $702(1.045 \%)$ patients were diagnosed with distant metastasis at initial presentation of DTC, including 323 men $(46.01 \%)$ and 379 women (53.99\%). BM accounts for $0.36 \%$ (244/67176) among entire group and 34.76\% (244/702) among subset with all distant metastasis in DTC patients. The sociodemographic, clinicopathological and treatment characteristics of population in this study were present in Table 1. Patterns of distant metastasis to specific sites in DTC was listed in Supplemental table 1 
Table 1

Clinical Characteristics of Patients with DTC with Identified BM at Diagnosis

Variable

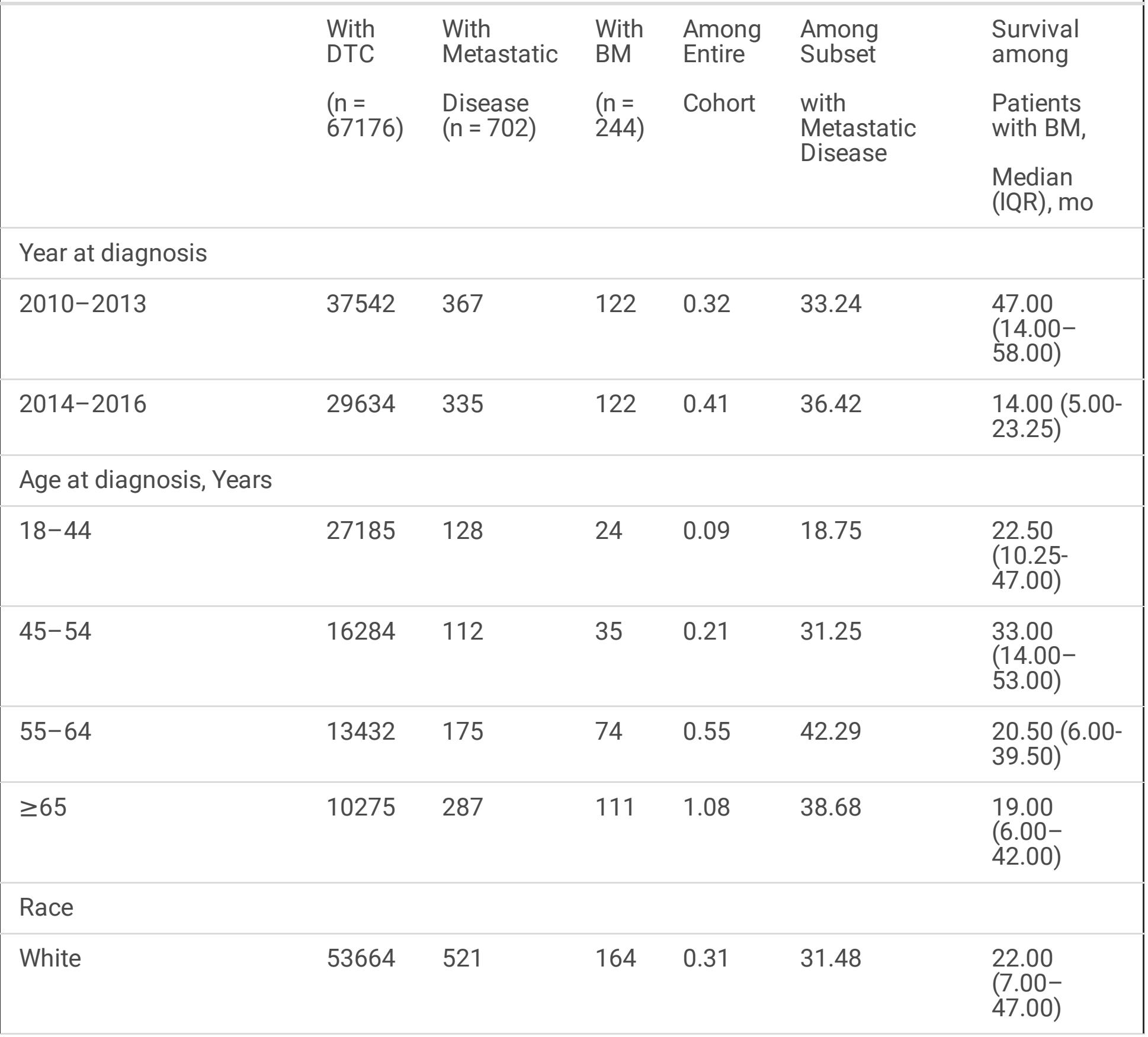

DTC, Differentiated Thyroid Carcinoma, BM, Bone Metastases, Cl, Confidence Interval, IQR, Interquartile Range, NA, Not Applicable.

† including American Indians, Alaska Natives and Asian-Pacific Islanders.

‡ divorced, separated, single (never married), and widowed

$\S$ according to the seventh edition of the AJCC Cancer Staging manual.

9 combination of beam with implants or isotopes 


\begin{tabular}{|c|c|c|c|c|c|c|}
\hline \multirow{2}{*}{$\begin{array}{l}\text { Variable } \\
\text { Black }\end{array}$} & \multicolumn{3}{|c|}{ Patients, No. } & \multicolumn{3}{|c|}{ Proportion of BM, \% } \\
\hline & 4685 & 61 & 35 & 0.75 & 57.38 & $\begin{array}{l}23.00 \\
(5.00- \\
35.00)\end{array}$ \\
\hline Otherst & 8827 & 120 & 45 & 0.51 & 37.5 & $\begin{array}{l}19.00(6.00- \\
46.50)\end{array}$ \\
\hline \multicolumn{7}{|l|}{ Gender } \\
\hline Male & 15085 & 323 & 103 & 0.68 & 31.89 & $\begin{array}{l}22.00 \\
(9.00- \\
48.00)\end{array}$ \\
\hline Female & 52091 & 379 & 141 & 0.27 & 37.2 & $\begin{array}{l}21.00 \\
(6.00- \\
43.00)\end{array}$ \\
\hline \multicolumn{7}{|c|}{ Insurance situation } \\
\hline Insured & 65472 & 674 & 236 & 0.36 & 35.01 & $\begin{array}{l}21.50 \\
(7.00- \\
47.00)\end{array}$ \\
\hline Uninsured & 1704 & 28 & 8 & 0.47 & 28.57 & $\begin{array}{l}33.00 \\
(4.25- \\
46.75)\end{array}$ \\
\hline \multicolumn{7}{|c|}{ Marital status } \\
\hline Married & 40859 & 381 & 138 & 0.34 & 36.22 & $\begin{array}{l}22.00 \\
(9.00- \\
48.00)\end{array}$ \\
\hline Unmarried $\neq$ & 22346 & 295 & 99 & 0.44 & 33.56 & $\begin{array}{l}21.00 \\
(5.00- \\
44.00)\end{array}$ \\
\hline Unkonwn & 3971 & 26 & 7 & 0.17 & 26.92 & $\begin{array}{l}17.00 \\
(17.00- \\
35.00)\end{array}$ \\
\hline
\end{tabular}

DTC, Differentiated Thyroid Carcinoma, BM, Bone Metastases, Cl, Confidence Interval, IQR, Interquartile Range, NA, Not Applicable.

† including American Indians, Alaska Natives and Asian-Pacific Islanders.

¥ divorced, separated, single (never married), and widowed

$\S$ according to the seventh edition of the AJCC Cancer Staging manual.

9 combination of beam with implants or isotopes 


\begin{tabular}{|c|c|c|c|c|c|c|}
\hline \multirow{2}{*}{$\begin{array}{l}\text { Variable } \\
\text { Unilateral }\end{array}$} & \multicolumn{3}{|c|}{ Patients, No. } & \multicolumn{3}{|c|}{ Proportion of BM, \% } \\
\hline & 66640 & 699 & 243 & 0.36 & 34.76 & $\begin{array}{l}22.00 \\
(7.00- \\
47.00)\end{array}$ \\
\hline Bilateral & 536 & 3 & 1 & 0.19 & 33.33 & $\begin{array}{l}22.00(7.00- \\
46.50)\end{array}$ \\
\hline \multicolumn{7}{|l|}{ Histologic } \\
\hline Papillary & 63945 & 582 & 161 & 0.25 & 27.66 & $\begin{array}{l}21.00(7.00- \\
41.50)\end{array}$ \\
\hline Follicular & 3231 & 120 & 83 & 2.57 & 69.17 & $\begin{array}{l}23.00 \\
(7.00- \\
49.00)\end{array}$ \\
\hline \multicolumn{7}{|c|}{ AJCC clinical stage§ } \\
\hline I & 49301 & 0 & 0 & 0 & NA & NA \\
\hline II & 4696 & 0 & 0 & 0 & NA & NA \\
\hline III & 9021 & 0 & 0 & 0 & NA & NA \\
\hline IV & 4158 & 702 & 244 & 5.87 & 34.76 & $\begin{array}{l}22.00 \\
(7.00- \\
47.00)\end{array}$ \\
\hline \multicolumn{7}{|c|}{ AJCC T classification§ } \\
\hline T1 & 40294 & 101 & 44 & 0.11 & 43.56 & $\begin{array}{l}25.50(6.50- \\
40.25)\end{array}$ \\
\hline T2 & 11168 & 82 & 42 & 0.38 & 51.22 & $\begin{array}{l}26.50 \\
(9.50- \\
51.50)\end{array}$ \\
\hline Т3 & 13838 & 263 & 91 & 0.66 & 34.6 & $\begin{array}{l}26.00 \\
(10.00- \\
53.00)\end{array}$ \\
\hline T4 & 1876 & 256 & 67 & 3.57 & 26.17 & $\begin{array}{l}11.00 \\
(5.00- \\
24.00)\end{array}$ \\
\hline \multicolumn{7}{|c|}{$\begin{array}{l}\text { DTC, Differentiated Thyroid Carcinoma, BM, Bone Metastases, Cl, Confidence Interval, IQR, } \\
\text { Interquartile Range, NA, Not Applicable. }\end{array}$} \\
\hline \multicolumn{7}{|c|}{ † including American Indians, Alaska Natives and Asian-Pacific Islanders. } \\
\hline \multicolumn{7}{|c|}{ ‡ divorced, separated, single (never married), and widowed } \\
\hline \multicolumn{7}{|c|}{$\S$ according to the seventh edition of the AJCC Cancer Staging manual. } \\
\hline
\end{tabular}




\section{Variable}

AJCC N classification§

NO

N1

51387

277

$15789 \quad 425$

$154 \quad 0.3$

$154 \quad 0.3$

55.6

Proportion of BM, \%

Patients, No.

$90 \quad 0.57$

21.18

NA

NA

MO

664740

702

702

244

34.76

34.76

22.00

(7.00-

47.00)

Multifocality

No

38843

337

128

0.33

37.98

$19.50(6.00-$ 43.50)

Yes

$28333 \quad 365$

$116 \quad 0.41$

31.78
22.00

$(8.50-$

47.00)

Extraskeletal

metastases to

lung, liver and brain, No.

\begin{tabular}{lllllll}
0 & 66734 & 260 & 141 & 0.21 & 54.23 & $\begin{array}{l}28.00 \\
(14.00- \\
53.00)\end{array}$ \\
1 & 82 & 82 & 82 & 100 & 100 & $\begin{array}{l}15.00(6.00- \\
29.50)\end{array}$ \\
2 & 36 & 36 & 21 & 100 & 58.33 & $\begin{array}{l}6.00(4.00- \\
8.50)\end{array}$ \\
3 & 1 & 1 & 0 & 100 & 0 & NA \\
\hline Unkonwn & 323 & 323 & 0 & 82.25 & 0 & NA
\end{tabular}

DTC, Differentiated Thyroid Carcinoma, BM, Bone Metastases, Cl, Confidence Interval, IQR, Interquartile Range, NA, Not Applicable.

† including American Indians, Alaska Natives and Asian-Pacific Islanders.

‡ divorced, separated, single (never married), and widowed

$\S$ according to the seventh edition of the AJCC Cancer Staging manual.

9 combination of beam with implants or isotopes 


\begin{tabular}{|c|c|c|c|c|c|c|}
\hline Variable & \multicolumn{3}{|c|}{ Patients, No. } & \multicolumn{3}{|c|}{ Proportion of BM, \% } \\
\hline \multicolumn{7}{|l|}{ Surgery } \\
\hline No & 568 & 87 & 38 & 6.69 & 43.68 & $\begin{array}{l}6.00(2.75- \\
22.50)\end{array}$ \\
\hline Lobectomy & 8904 & 33 & 10 & 0.11 & 30.3 & $\begin{array}{l}16.00(4.00- \\
23.25)\end{array}$ \\
\hline $\begin{array}{l}\text { Subtotal or near-total } \\
\text { thyroidectomy }\end{array}$ & 1758 & 18 & 1 & 0.06 & 5.56 & NA \\
\hline Total thyroidectomy & 55946 & 564 & 195 & 0.35 & 34.57 & $\begin{array}{l}25.00 \\
(10.00- \\
50.00)\end{array}$ \\
\hline \multicolumn{7}{|l|}{ Radiation therapy } \\
\hline No & 36712 & 171 & 43 & 0.11 & 25.15 & $\begin{array}{l}7.00(3.00- \\
41.00)\end{array}$ \\
\hline $\begin{array}{l}\text { Radiation beam or } \\
\text { radioactive implants }\end{array}$ & 1070 & 163 & 88 & 8.22 & 53.99 & $\begin{array}{l}11.00(5.00- \\
30.25)\end{array}$ \\
\hline Radioisotopes & 29293 & 348 & 97 & 0.33 & 27.87 & $\begin{array}{l}27.00 \\
(17.00- \\
54.00)\end{array}$ \\
\hline Combination 9 & 100 & 20 & 16 & 16 & 80 & $\begin{array}{l}35.00 \\
(20.50- \\
59.00)\end{array}$ \\
\hline \multicolumn{7}{|l|}{ Chemotherapy } \\
\hline No & 66990 & 638 & 224 & 0.33 & 35.11 & $\begin{array}{l}22.00 \\
(8.00- \\
47.00)\end{array}$ \\
\hline Yes & 186 & 64 & 20 & 10.75 & 31.25 & $\begin{array}{l}7.00(3.25- \\
24.00)\end{array}$ \\
\hline \multicolumn{7}{|c|}{$\begin{array}{l}\text { DTC, Differentiated Thyroid Carcinoma, BM, Bone Metastases, Cl, Confidence Interval, IQR, } \\
\text { Interquartile Range, NA, Not Applicable. }\end{array}$} \\
\hline \multicolumn{7}{|c|}{ † including American Indians, Alaska Natives and Asian-Pacific Islanders. } \\
\hline \multicolumn{7}{|c|}{ ‡ divorced, separated, single (never married), and widowed } \\
\hline \multicolumn{7}{|c|}{$\S$ according to the seventh edition of the AJCC Cancer Staging manual. } \\
\hline 9 combination of bear & h implan & or iso & & & & \\
\hline
\end{tabular}


Trends in DTC patients with specific distant metastasis are described in Supplemental table 2 and Fig. 2, with joinponit regression denoted as trends 1 to 2. Incidence rates of BM in DTC patients remained relatively stable during the study period (from 1.23 [95\% Cl, 0.91 to 1.62] per 1000000 person-years in 2010 to 1.36 [ $95 \% \mathrm{Cl}, 1.06$ to 1.74 per 1000000 person-years in 2016), with an AAPC of $2.52(95 \% \mathrm{Cl}$, -2.81 to $8.14, P=0.28$ ). Incidence rates of other specific distant metastasis including sites to lung, brain and livers also have been stable in recent years.

\section{Predictors of BM in DTC patients}

On Chi-square or Fisher's test, age at diagnosis, gender, race, marital status, histology, AJCC T classification, AJCC N classification, surgery, radiation therapy and chemotherapy showed significant difference among the entire cohort (Supplemental table 3). Within the entire cohort, proportion of older patients (age $\geq 65$ years old) $(45.4 \%$ vs $15.2 \%, P<0.001)$, black race $(14.3 \%$ vs $6.9 \%, P<0.001)$, male gender ( $42.2 \%$ vs $22.4 \%, \mathrm{P}<0.001)$, unmarried patients $(40.6 \%$ vs $33.2 \%, \mathrm{P}=0.014)$, follicular histology (34.0\% vs $4.7 \%, \mathrm{P}<0.001)$, AJCC T4 staging ( $27.5 \%$ vs $2.7 \%, \mathrm{P}<0.001)$, AJCC N1 staging $(36.9 \%$ vs $23.5 \%, \mathrm{P}<0.001)$, no surgery $(15.6 \%$ vs $0.8 \%, \mathrm{P}<0.001)$, radiation beam or radioactive implants $(36.1 \%$ vs $1.5 \%, P<0.001)$, chemotherapy ( $8.2 \%$ vs $0.2 \%, P<0.001$ ) were associated with $B M$. Of note, $P$ value of AJCC clinical stage between two groups was not calculatable because all BM patients were in AJCC 7th stage IV.

Univariable logistic regression in the entire cohort figured out 10 statistically significant $(P<0.05)$ factors, including the age at diagnosis, race, gender, marital status, histology, AJCC T classification, AJCC N classification, surgery, radiation therapy and chemotherapy (Supplemental table 4). All these statistically significant factors were entered into the multivariable logistic regression. The multivariable logistic regression model of the predictors of presence of BM in DTC patients was constructed in Table 2. Age between 45 to 54 years (vs age between 18 to 44 years; OR: $2.562,95 \% \mathrm{Cl}: 1.500$ to $4.374, \mathrm{P}=0.001$ ), age between 55 to 64 years (vs age between 18 to 44 years; OR: $5.644,95 \% \mathrm{Cl}: 3.485$ to $9.140, \mathrm{P}<0.001$ ), age $\geq 65$ years (vs age between 18 to 44 years; OR: $7.370,95 \% \mathrm{Cl}: 4.589$ to $11.838, \mathrm{P}<0.001$ ), black race (vs white race; OR: $2.538,95 \% \mathrm{Cl}: 1.675$ to $3.847, \mathrm{P}<0.001$ ), follicular histology (vs papillary histology; OR: 8.141, 95\%Cl: 5.809 to $11.409, \mathrm{P}<0.001$ ), AJCC T2 staging (vs AJCC T1 staging; OR: $1.624,95 \% \mathrm{Cl}: 1.032$ to 2.557, $\mathrm{P}=0.036$ ), AJCC T3 staging (vs AJCC T1 staging; OR: $2.354,95 \% \mathrm{Cl}: 1.582$ to $3.503, \mathrm{P}<0.001$ ), AJCC T4 staging (vs AJCC T1 staging; OR: $3.595,95 \% \mathrm{Cl}: 2.169$ to $5.958, \mathrm{P}<0.001$ ), radiation beam or radioactive implants (vs no radiation therapy; OR: $25.477,95 \% \mathrm{Cl}: 16.469$ to $39.411, \mathrm{P}<0.001$ ), radioisotopes (vs no radiation therapy; OR: $2.097,95 \% \mathrm{Cl}: 1.420$ to $3.097, \mathrm{P}<0.001$ ), Combination radiation (vs no radiation therapy; OR: $70.766,95 \% \mathrm{Cl}: 34.842$ to $143.727, \mathrm{P}<0.001$ ), chemotherapy (vs no chemotherapy; OR: $1.920,95 \% \mathrm{Cl}: 1.008$ to $3.656, \mathrm{P}<0.001)$ could serve as significant predictors of presenting BM in newly diagnosed DTC. Meanwhile, female gender (vs male gender; OR: $0.721,95 \% \mathrm{Cl}$ : 0.540 to $0.963, P=0.027$ ), lobectomy (vs no surgery; OR: $0.071,95 \% \mathrm{Cl}: 0.032$ to $0.160, \mathrm{P}<0.001$ ), subtotal or near-total thyroidectomy (vs no surgery; OR: $0.018,95 \% \mathrm{Cl}: 0.002$ to $0.144, \mathrm{P}<0.001$ ), total thyroidectomy (vs no surgery; OR: $0.164,95 \% \mathrm{Cl}$ : 0.096 to $0.279, \mathrm{P}<0.001$ ) were significantly associated with lower possibility of BM in newly diagnosed DTC. 
Table 2

Multivariable Logistic Regression for the Presence of BM at Diagnosis of DTC

\section{Variable}

Age at diagnosis, Years

Among entire cohort

Among subset with

metastatic disease

OR $(95 \% \mathrm{Cl})$

P
Value

OR $(95 \% \mathrm{Cl})$

NA

0.001

2.562 (1.500-4.374)

$5.644(3.485-9.140)$

$7.370(4.589-$

11.838)

Reference
$2.562(1.500-4.374)$
$5.644(3.485-9.140)$
$7.370(4.589-$
$11.838)$

Reference

$\begin{array}{lll}< & 2.619(1.424- & 0.002 \\ 0.001 & 4.817) & \\ < & \begin{array}{l}1.865(1.034- \\ 0.001\end{array} & 3.365)\end{array}$

Race

White

2.538 (1.675-3.847)

NA

Reference

NA

Black

$1.787(1.242-2.571)$

<. 001

1.916 (1.003-
$3.662)$

0.049

Otherst

0.002

$1.053(0.650-$
$1.704)$

0.835

Gender

Male

Reference

NA

NA

NA

Female

$0.721(0.540-0.963)$

0.027

NA

NA

Marital status

Married

Reference

NA

NA

NA

Unmarrited $\neq$

1.202 (0.897-1.611)

0.218

NA

NA

Unkonwn

0.579 (0.254-1.320)

$0.194 \quad N A$

NA

DTC, Differentiated Thyroid Carcinoma, BM, Bone Metastases, OR, odds ratio, Cl, Confidence Interval, NA, Not Applicable.

† including American Indians, Alaska Natives and Asian-Pacific Islanders.

₹ divorced, separated, single (never married), and widowed

$\S$ according to the seventh edition of the AJCC Cancer Staging manual.

9 combination of beam with implants or isotopes 
Variable

Among entire cohort

Among subset with

metastatic disease

Histologic

Papillary

Follicular

AJCC T classification§

$\mathrm{T} 1$

T2

T3

T4

AJCC N classification§

NO

N1

Reference

$1.312(0.934-1.844)$
Reference

8.141 (5.809-

11.409)
NA

$<$

0.001
Reference

3.237 (1.962-

$5.340)$

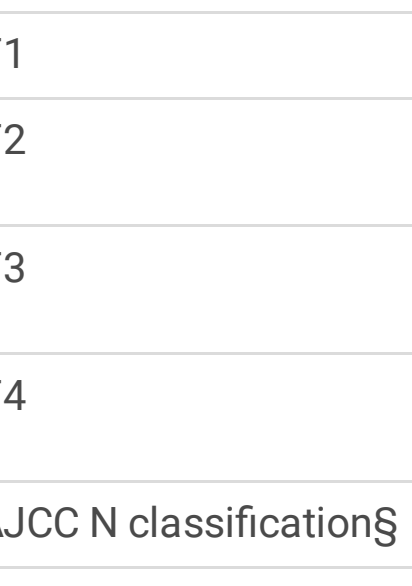

1

Reference

NA

Reference

1.624 (1.032-2.557)

0.036

$1.177(0.585-$

2.370)

$2.354(1.582-3.503)$

<.001

$0.805(0.465-$

$\begin{array}{ll}3.595(2.169-5.958) & < \\ 0.001\end{array}$

$0.495(0.272-0.900)$
NA

$<$

0.001

NA

0.648

0.44

0.021

Surgery

No

Reference

NA

Reference

NA

Lobectomy

$0.071(0.032-0.160)$

$\begin{array}{ll}< & 0.695(0.261- \\ 0.001 & 1.850)\end{array}$

Subtotal or near-total

thyroidectomy

$0.018(0.002-0.144)$

$<$

0.001

$0.043(0.005-$

$0.397)$

Total thyroidectomy

$\begin{array}{lll}0.164(0.096-0.279) & < & 0.743(0.409- \\ & 0.001 & 1.348)\end{array}$

$0.743(0.41$
$1.348)$

Radiation therapy

DTC, Differentiated Thyroid Carcinoma, BM, Bone Metastases, OR, odds ratio, Cl, Confidence Interval, NA, Not Applicable.

† including American Indians, Alaska Natives and Asian-Pacific Islanders.

‡ divorced, separated, single (never married), and widowed

$\S$ according to the seventh edition of the AJCC Cancer Staging manual.

9 combination of beam with implants or isotopes 


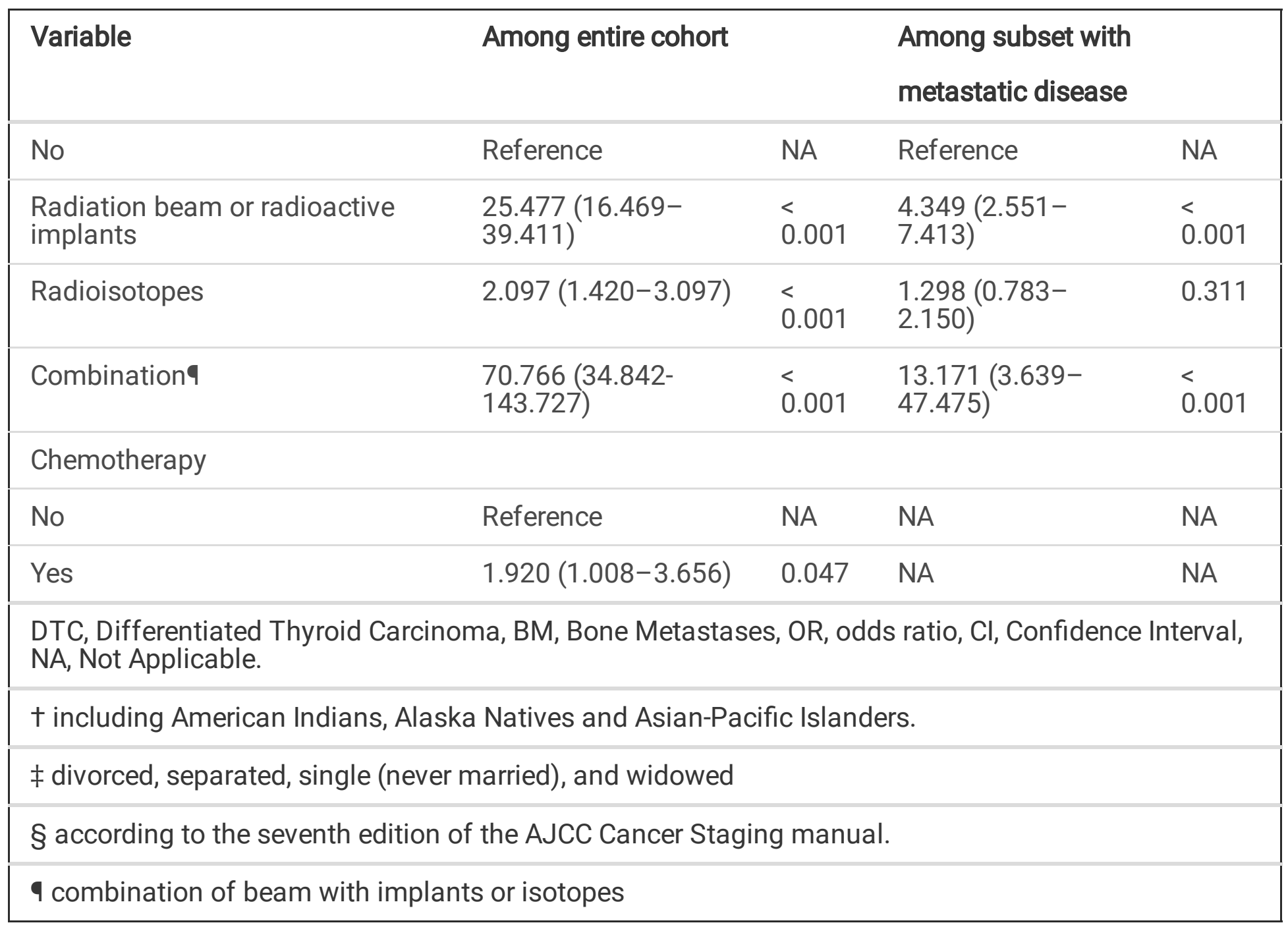

Moreover, ROC analysis was conducted to further assess the multivariable logistic regression models with significant predictors above. The multivariable logistic regression model of the entire cohort exhibited optimal performance with the value of AUC of 0.893 (Supplemental Fig. 1). Furthermore, the nomogram predicting the probability of BM in DTC patients was further established based on significant predictors identified through multivariable logistic regression (Fig. 3). Each variable had a corresponding score in the nomogram (Supplemental table 5). We could estimate the probability of undergoing amputation according to the total scores.

\section{Survival Analysis}

Findings above gave insight of BM incidence and prediction of presence in newly diagnosed DTC patients. In order to explore roles of these factors in prognosis of DTC patients with BM, we carried out the survival analysis. The univariable cox regression analysis for overall survival and cancer-specific survival in DTC patients with BM was present in Table 3. On multivariable analysis (Table 4) among the subset with $\mathrm{BM}$, we identified three factors that were significantly associated with overall survival, including metastatic sites $\geq 2$ (vs metastatic sites $<2$; OR: $2.394,95 \% \mathrm{Cl}: 1.376$ to $4.164, \mathrm{P}=0.002$ ), total thyroidectomy (vs no surgery; OR: $0.388,95 \% \mathrm{Cl}: 0.224$ to $0.673, \mathrm{P}=0.001$ ), radioisotopes (vs no radiation 
therapy; OR: $0.451,95 \% \mathrm{Cl}: 0.216$ to $0.941, \mathrm{P}=0.034)$. And factors linked with cancer-specific survival were diagnosis at 2014-2016 (vs diagnosis at 2010-2013; OR: $0.494,95 \% \mathrm{Cl}: 0.270$ to $0.904, \mathrm{P}=0.022$ ), metastatic sites $\geq 2$ (vs metastatic sites < 2; OR: 4.366, 95\%Cl: 2.259 to $8.435, \mathrm{P}<0.001$ ), total thyroidectomy (vs no surgery; OR: $0.381,95 \% \mathrm{Cl}: 0.202$ to $0.720, \mathrm{P}=0.003$ ). Overall survival estimates as stratified by metastatic site (Fig. 4A), surgery (Fig. 4B) and radiation therapy (Fig. 4C), and cancer-specific survival estimates as stratified by metastatic site (Fig. 4D), surgery (Fig. 4E), year at diagnosis (Fig. 4F) were illustrated in the Fig. 4. 
Table 3

Univariable Analysis for Overall and Cancer-specific Survival in Patients with DTC diagnosed with BM

Variable

HR( $95 \%$ Cl)

Year at diagnosis

$2010-2013$

2014-2016

Age at diagnosis, Years

$18-44$

$45-54$

$55-64$

$\geq 65$

Race

White

Black

Otherst

Gender

Male

Female

Insurance situation

1.227 (0.497-

3.028)

1.685 (0.718-

3.954)

Reference

$1.226(0.669-$

2.247)

0.804 (0.430-

1.502)

Reference

$1.014(0.654-$
Overall Survival

Reference

0.733 (0.437-

1.230)
P

Value
Cancer-specific Survival

$\mathrm{HR}(95 \% \mathrm{Cl})$

Value

NA Reference

NA

0.24

$0.616(0.348-$

1.089)

0.095

Reference NA Reference NA

$\begin{array}{llll}0.642(0.215- & 0.427 & 0.471(0.126- & 0.262 \\ 1.916) & & 1.756)\end{array}$

0.657

1.352 (0.507-

0.546

3.605)

0.23

1.657 (0.650-

4.225)

NA Reference

NA

0.51

0.978 (0.479-

0.952

1.997)

0.493

0.704 (0.345-

1.437)

0.335

NA

Reference

NA

0.95

1.122 (0.685-

1.838)

0.649

DTC, Differentiated Thyroid Carcinoma, BM, Bone Metastases, HR, hazard ratio, Cl, Confidence Interval, NA, Not Applicable.

† including American Indians, Alaska Natives and Asian-Pacific Islanders.

‡ divorced, separated, single (never married), and widowed

$\S$ according to the seventh edition of the AJCC Cancer Staging manual.

9 combination of beam with implants or isotopes 


\begin{tabular}{|c|c|c|c|c|}
\hline \multirow{2}{*}{$\begin{array}{l}\text { Variable } \\
\text { Insured }\end{array}$} & \multicolumn{2}{|l|}{ Overall Survival } & \multicolumn{2}{|c|}{ Cancer-specific Survival } \\
\hline & Reference & NA & Reference & NA \\
\hline Uninsured & $\begin{array}{l}1.577(0.576- \\
4.317)\end{array}$ & 0.375 & $\begin{array}{l}0.951(0.233- \\
3.890)\end{array}$ & 0.945 \\
\hline \multicolumn{5}{|l|}{ Marital status } \\
\hline Married & Reference & NA & Reference & NA \\
\hline Unmarrited $\neq$ & $\begin{array}{l}1.350(0.866- \\
2.103)\end{array}$ & 0.185 & $\begin{array}{l}1.245(0.759- \\
2.041)\end{array}$ & 0.385 \\
\hline Unkonwn & $\begin{array}{l}0.516(0.071- \\
3.753)\end{array}$ & 0.513 & $\begin{array}{l}0.601(0.082- \\
4.390)\end{array}$ & 0.615 \\
\hline \multicolumn{5}{|l|}{ Histologic } \\
\hline Papillary & Reference & NA & Reference & NA \\
\hline Follicular & $\begin{array}{l}0.884(0.553- \\
1.413)\end{array}$ & 0.607 & $\begin{array}{l}0.954(0.570- \\
1.595)\end{array}$ & 0.856 \\
\hline \multicolumn{5}{|c|}{ AJCC T classification§ } \\
\hline T1 & Reference & NA & Reference & NA \\
\hline T2 & $\begin{array}{l}0.952(0.393- \\
2.305)\end{array}$ & 0.913 & $\begin{array}{l}0.945(0.330- \\
2.692)\end{array}$ & 0.912 \\
\hline T3 & $\begin{array}{l}1.016(0.484- \\
2.133)\end{array}$ & 0.966 & $\begin{array}{l}1.134(0.476- \\
2.702)\end{array}$ & 0.776 \\
\hline $\mathrm{T} 4$ & $\begin{array}{l}3.020(1.488- \\
6.126)\end{array}$ & 0.002 & $\begin{array}{l}3.478(1.533- \\
7.889)\end{array}$ & 0.003 \\
\hline \multicolumn{5}{|c|}{ AJCC N classification§ } \\
\hline NO & Reference & NA & Reference & NA \\
\hline $\mathrm{N} 1$ & $\begin{array}{l}1.904(1.226- \\
2.956)\end{array}$ & 0.004 & $\begin{array}{l}2.020(1.240- \\
3.290)\end{array}$ & 0.005 \\
\hline
\end{tabular}

DTC, Differentiated Thyroid Carcinoma, BM, Bone Metastases, HR, hazard ratio, $\mathrm{Cl}$, Confidence Interval, NA, Not Applicable.

† including American Indians, Alaska Natives and Asian-Pacific Islanders.

¥ divorced, separated, single (never married), and widowed

$\S$ according to the seventh edition of the AJCC Cancer Staging manual.

9 combination of beam with implants or isotopes 


\begin{tabular}{|c|c|c|c|c|}
\hline \multirow{2}{*}{$\begin{array}{l}\text { Variable } \\
\text { No }\end{array}$} & \multicolumn{2}{|l|}{ Overall Survival } & \multicolumn{2}{|c|}{ Cancer-specific Survival } \\
\hline & Reference & NA & Reference & NA \\
\hline Yes & $\begin{array}{l}4.014(2.510- \\
6.402)\end{array}$ & $<.001$ & $\begin{array}{l}6.224(3.512- \\
11.028)\end{array}$ & $\begin{array}{l}< \\
0.001\end{array}$ \\
\hline \multicolumn{5}{|l|}{ Multifocality } \\
\hline No & Reference & NA & Reference & NA \\
\hline Yes & $\begin{array}{l}0.903(0.581- \\
1.402)\end{array}$ & 0.649 & $\begin{array}{l}0.746(0.454- \\
1.220)\end{array}$ & 0.242 \\
\hline \multicolumn{5}{|l|}{ Surgery } \\
\hline No & Reference & NA & Reference & NA \\
\hline Lobectomy & $\begin{array}{l}0.987(0.423- \\
2.303)\end{array}$ & 0.977 & $\begin{array}{l}1.181(0.494- \\
2.824)\end{array}$ & 0.708 \\
\hline $\begin{array}{l}\text { Subtotal or near-total } \\
\text { thyroidectomy }\end{array}$ & $\begin{array}{l}8.427(1.058- \\
67.143)\end{array}$ & 0.044 & $\begin{array}{l}8.871(1.106- \\
71.166)\end{array}$ & 0.04 \\
\hline Total thyroidectomy & $\begin{array}{l}0.205(0.125- \\
0.335)\end{array}$ & $<0.001$ & $\begin{array}{l}0.218(0.124- \\
0.380)\end{array}$ & $<.001$ \\
\hline \multicolumn{5}{|l|}{ Radiation therapy } \\
\hline No & Reference & NA & Reference & NA \\
\hline $\begin{array}{l}\text { Radiation beam or radioactive } \\
\text { implants }\end{array}$ & $\begin{array}{l}1.311(0.747- \\
2.300)\end{array}$ & 0.346 & $\begin{array}{l}1.206(0.646- \\
2.253)\end{array}$ & 0.557 \\
\hline Radioisotopes & $\begin{array}{l}0.279(0.141- \\
0.553)\end{array}$ & $<0.001$ & $\begin{array}{l}0.301(0.143- \\
0.633)\end{array}$ & 0.002 \\
\hline Combination & $\begin{array}{l}0.277(0.081- \\
0.947)\end{array}$ & 0.041 & $\begin{array}{l}0.345(0.099- \\
1.203)\end{array}$ & 0.095 \\
\hline \multicolumn{5}{|l|}{ Chemotherapy } \\
\hline No & Reference & NA & Reference & NA \\
\hline Yes & $\begin{array}{l}3.756(2.051- \\
6.876)\end{array}$ & $<.001$ & $\begin{array}{l}4.076(2.156- \\
7.706)\end{array}$ & $\begin{array}{l}< \\
0.001\end{array}$ \\
\hline \multicolumn{5}{|c|}{$\begin{array}{l}\text { DTC, Differentiated Thyroid Carcinoma, BM, Bone Metastases, HR, hazard ratio, Cl, Confidence } \\
\text { Interval, NA, Not Applicable. }\end{array}$} \\
\hline \multicolumn{5}{|c|}{ † including American Indians, Alaska Natives and Asian-Pacific Islanders. } \\
\hline \multicolumn{5}{|c|}{ ‡ divorced, separated, single (never married), and widowed } \\
\hline \multicolumn{5}{|c|}{$\S$ according to the seventh edition of the AJCC Cancer Staging manual. } \\
\hline 9 combination of beam with im & or isotopes & & & \\
\hline
\end{tabular}


Page 18/31 
Table 4

Multivariable Analysis for Overall and Cancer-specific Survival in Patients with DTC diagnosed with BM

Variable

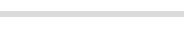

Overall Survival

$\mathrm{HR}(95 \% \mathrm{Cl})$

NA

NA

2014-2016

AJCC T classification§

$\begin{array}{lllll}\text { T1 } & \text { Reference } & \text { NA } & \text { Reference } & \text { NA } \\ \text { T2 } & 0.685(0.273- & 0.419 & 0.688(0.233- & 0.499 \\ & 1.716) & & 2.031) & \\ \text { T3 } & 0.684(0.312- & 0.343 & 0.596(0.240- & 0.264 \\ & 1.500) & & 1.477) & \\ \text { T4 } & 1.029(0.439- & 0.947 & 0.819(0.308- & 0.69 \\ & 2.413) & & 2.180) & \end{array}$

AJCC N classification§

NO

Reference

NA

Reference

NA

N1

$1.158(0.701-$

1.913)

0.566

1.422 (0.809-

2.498)
Cancer-specific Survival

$\mathrm{P}$

Value

NA

0.022

$0.904)$

Metastatic sites $\geq 2$

$\begin{array}{lllll}\text { No } & \text { Reference } & \text { NA } & \text { Reference } & \text { NA } \\ \text { Yes } & 2.394(1.376- & 0.002 & 4.366(2.259- & < \\ & 4.164) & & 8.435) & 0.001\end{array}$

Surgery

No

\begin{tabular}{|c|c|c|c|}
\hline Reference & NA & Reference & NA \\
\hline $\begin{array}{l}1.036(0.430- \\
2.498)\end{array}$ & 0.937 & $\begin{array}{l}0.945(0.367- \\
2.433)\end{array}$ & 0.907 \\
\hline $\begin{array}{l}3.428(0.406- \\
28.976)\end{array}$ & 0.258 & $\begin{array}{l}2.131(0.241- \\
18.813)\end{array}$ & 0.496 \\
\hline
\end{tabular}

Subtotal or near-t
thyroidectomy

DTC, Differentiated Thyroid Carcinoma, BM, Bone Metastases, HR, hazard ratio, $\mathrm{Cl}$, Confidence Interval, NA, Not Applicable.

$\S$ according to the seventh edition of the AJCC Cancer Staging manual.

9 combination of beam with implants or isotopes 


\begin{tabular}{|c|c|c|c|c|}
\hline \multirow{2}{*}{$\begin{array}{l}\text { Variable } \\
\text { Total thyroidectomy }\end{array}$} & \multicolumn{2}{|l|}{ Overall Survival } & \multicolumn{2}{|c|}{ Cancer-specific Survival } \\
\hline & $\begin{array}{l}0.388(0.224- \\
0.673)\end{array}$ & 0.001 & $\begin{array}{l}0.381(0.202- \\
0.720)\end{array}$ & 0.003 \\
\hline \multicolumn{5}{|l|}{ Radiation therapy } \\
\hline No & Reference & NA & Reference & NA \\
\hline $\begin{array}{l}\text { Radiation beam or radioactive } \\
\text { implants }\end{array}$ & $\begin{array}{l}1.318(0.740- \\
2.345)\end{array}$ & 0.348 & $\begin{array}{l}1.064(0.560- \\
2.023)\end{array}$ & 0.849 \\
\hline Radioisotopes & $\begin{array}{l}0.451(0.216- \\
0.941)\end{array}$ & 0.034 & $\begin{array}{l}0.537(0.238- \\
1.209)\end{array}$ & 0.133 \\
\hline Combination 9 & $\begin{array}{l}0.403(0.114- \\
1.427)\end{array}$ & 0.159 & $\begin{array}{l}0.534(0.143- \\
1.988)\end{array}$ & 0.349 \\
\hline \multicolumn{5}{|l|}{ Chemotherapy } \\
\hline No & Reference & NA & Reference & NA \\
\hline Yes & $\begin{array}{l}1.523(0.783- \\
2.963)\end{array}$ & 0.215 & $\begin{array}{l}1.786(0.866- \\
3.684)\end{array}$ & 0.116 \\
\hline \multicolumn{5}{|c|}{$\begin{array}{l}\text { DTC, Differentiated Thyroid Carcinoma, BM, Bone Metastases, HR, hazard ratio, Cl, Confidence } \\
\text { Interval, NA, Not Applicable. }\end{array}$} \\
\hline \multicolumn{5}{|c|}{$\S$ according to the seventh edition of the AJCC Cancer Staging manual. } \\
\hline 9 combination of beam with im! & or isotopes & & & \\
\hline
\end{tabular}

\section{Discussion}

Incidence of BM in DTC was relatively lower compared with that in other malignant cancers such lung cancer[19] and breast cancer[20,21]. Besides, BM often occurs in medullary and undifferentiated thyroid cancer[22], and limited characteristics about BM in DTC have been reported. Despite several case reports and cohort studies[23-25], little was known about its incidence, risk factor and prognosis in DTC. In this population-based study, we analyzed the trend in incidence, survival, risk factors and prognostic factors of BM in newly diagnosed DTC patients, aiming to promote understanding on the current situation and the association between clinical pattern and BM. To the best of our knowledge, this study included largest sample size of DTC patients with BM compared with previous ones.

In the present study utilizing SEER database, BM accounts for $0.36 \%(244 / 67176)$ among entire group and $34.76 \%$ (244/702) among subset with metastatic diseases in newly diagnosed DTC patients, which was lower than the proportion (2.0\%) in study of Yorihisa et al.[9] and the proportion (5.51\%) in study of Marie-odile et al.[26] Sample size in these two studies was relatively small and they only included patients in a single institution; therefore the results in the above studies were not representative. In 
addition, the above two studies were conducted with date in early period, while our study focused on patients diagnosed during 2010 to 2016. With the advancement of screening methods, early detection of asymptomatic thyroid cancer and timely interventions such as surgery have substantially enhanced the prognosis of thyroid tumor patients. Therefore, the frequency of bone metastases in DTC patients may have reduced compared with early decades.

To explore the trend of incidence of BM in DTC patients, Jointpoint regression analysis was introduced, and result indicated that the age-adjusted incidence of BM in DTC was relatively stable during study period, which was not reported before. Besides, incidence of thyroid cancer was in constant increasing during 1974 to 2013 in United States[1].

By using multivariable logistic regression, we recognized predictors of BM in newly diagnosed DTC. Older age, black race, male gender, follicular histology, more advanced T stage, radiation therapy, no surgery, and chemotherapy were significantly associated with of presence of BM in DTC patients at diagnosis. In a meta-analysis that enrolled 34 articles with 73,219 patients, age $\geq 45$ years, male gender, and follicular histology were demonstrated to be significant predictors of distant metastasis, which was consistent with our analysis [27]. Follicular thyroid cancer was characterized with higher invasion and distant metastasis compared with papillary thyroid cancer; therefore BM rate was higher correspondently[28]. According to a population analysis included patients with DTC during 1988-2009 in SEER database, patients with distant metastasis tended to have received radiation therapy and not to have had surgery[29]. However, as it was discussed, SEER database did not provide information about specific anatomic sites of distant metastasis at that time, so the detailed data for BM was not getable in their analysis.

Furthermore, the optimal performance of multivariable logistic regression model in the entire cohort was authenticated by ROC analysis, in which the AUC value was 0.893 . Besides, the nomogram established in this study is an efficient tool to help doctors decision-making owing to its user-friendly interface and optimal predictive ability. Nevertheless, we were not capable of figuring out the most common site for BM because there was no data documented in SEER database until now.

Prognostic factors for overall survival and cancer-specific survival of DTC patient with BM was analyzed using the multivariable Cox model. Results revealed that patients with multiple metastatic sites have higher hazard ratio for overall survival and cancer-specific survival, which was in coherence with previous studies[10,30]. Meanwhile, significant decrease in the hazard ratio for overall survival and cancer-specific survival was found in patients with total thyroidectomy and radioisotopes radiation therapy. Surgery was the mainstay of therapy for thyroid cancer patients, especially in patients without distant metastasis[31]. Surgical options include thyroid total thyroidectomy or lobectomy, and it was still in controversy on how to choose optimal surgical procedure because of the difficulty in balancing surgical effect and complication[32]. Total thyroidectomy reduces recurrence and allows early detection of recurrence in the neck using ultrasonography compared with lobectomy, thereafter improving the prognosis compared with others. The most used radioisotope in DTC was radioiodine, but its using was still of wide divergence, partially due to the lack of evident prospective randomized controlled trials[33]. Patients with BM could 
benefit from radioiodine in both suppressing the progression of tumor and reducing recurrence of primary carcinoma as we concluded. It was testified in some retrospective studies that application of radioiodine could improve survival of DTC patients[9, 34,35] as well as BM patients[36]. Especially, radioiodine therapy was effective in the ablation of thyroid remnant after surgery[37]. External beam radiation therapy (EBRT) was not associated with positive outcomes in our analysis. Inversely, a retrospective study enrolled 74 BM patients has demonstrated that EBRT significantly increased survival[38]. Nevertheless, EBRT was reported to have acute toxicities such as esophageal stricture, so it was used under relatively strict indication[39]. Besides, combining radioiodine and external beam radiation therapy seems advantageous in some cases[40]. However, the treatment effect of total thyroidectomy and radioiodine in DTC patients with BM need to be further explored in the future study. Other factors did not exhibit prognostic value in our analysis, but their role has been demonstrated in other studies. Older age, higher T stage, positive lymph node, and black race were predictors of positive outcome[24, 25]. Further study is necessary to shed light on their prognostic value in BM.

Although this was a population-based study that enrolled largest cohort of DTC patients with BM, limitations in this study was unneglectable. Because it was a retrospective study based on current database record, some parameters were unavailable. Firstly, the further detailed information of BM like specific sites was not available in SEER database, which limited further analysis. Secondly, onset of BM during follow-up period was not presented and we only had information on synchronous metastasis, so the incidence may be underestimated. Thirdly, more detailed information about radiation or chemotherapy such as chemotherapy regimens, doses, and the specific number of cycles were not recorded in SEER database. Fourthly, except the survival, other outcomes such as complications of exposures or interventions were not reported. We could only assess the prognosis by survival data. Finally, data on specific metastatic sites including lung, bone, liver and brain was available in SEER database since 2010 , leading to some inevitable bias. Therefore, more convincible clinical studies especially the randomized clinical trials were necessitated in the future.

In summary, results of this population-based study highlighted the incidence, predictors and prognostic factors of BM in newly diagnosed DTC patients. The age-adjusted incidence of BM in DTC was relatively stable during study period. Older age, male gender, black race, follicular histology, more advanced T staging, no surgery, radiation therapy, and chemotherapy were significant predictors for the presence BM in DTC patients. Thus, timely, and appropriate screenings for patients with these risk factors are recommended. Moreover, in DTC patients with BM, presence of total thyroidectomy and radioisotopes radiation therapy exhibited significant benefit on overall survival (OS) and cancer-specific survival (CSS) while multiple metastatic sites could serve as indicator of poor prognosis, which provided evidence for clinical practice.

\section{List Of Abbreviations}

DM: distant metastasis 
DTC: differentiated thyroid carcinoma

BM: bone metastasis

SEER: Surveillance, Epidemiology, and End Results

ROC: receiver operating characteristic

APCC: average annual percentage change

AUC: value of area under curve

AJCC: American joint committee on cancer

APC: annual percentage change

OR: odds ratio

Cl: confidence intervals

EBRT: external beam radiation therapy

OS: overall survival

CSS: cancer-specific survival

\section{Declarations}

Ethics approval and consent to participate

Not applicable

\section{Consent to publication}

Not applicable

\section{Availability of data and materials}

The datasets generated and/or analyzed during the current study are available in the [Surveillance, Epidemiology, and End Results (SEER)] repository, [seer.cancer.gov].

\section{Competing interests}

Zhihong Li and Chao Tu are members of the editorial board of BMC cancer. Other authors declare that they have no competing interests.

\section{Funding}


This study was supported by Natural Science Foundation of Hunan Province, China (2018JJ3716), National Natural Science Foundation of China (81902745), and the Hunan Provincial Science and Technology Association program(2017TJ-Q19).

\section{Authors' contributions}

LQ and WCZ conceived and designed the work. Material preparation, data collection and analysis were performed by LQ, WCZ, XLR, and CQL. The first draft of the manuscript was written by LQ and WCZ, and all authors commented on previous versions of the manuscript. CT and ZHL contributed to article drafting, critical revision and final approval of the version to be published. All authors read and approved the final manuscript.

\section{Acknowledgements}

Not applicable

\section{Authors' Information}

L.Qi's e-mail address: qi.lin@csu.edu.cn

W.Zhang's e-mail address: 2204140314@csu.edu.cn

X.Ren's e-mail address: 2204120114@csu.edu.cn

C.Liu's e-mail address: liuchaoqian2002@aliyun.com

C.Tu's e-mail address: tuchao@csu.edu.cn

Z.Li's e-mail address: lizhihong@csu.edu.cn

\section{References}

1. Lim H, Devesa SS, Sosa JA, Check D, Kitahara CM: Trends in Thyroid Cancer Incidence and Mortality in the United States, 1974-2013. JAMA 2017, 317(13):1338-1348.

2. Bray F, Ferlay J, Soerjomataram I, Siegel RL, Torre LA, Jemal A: Global cancer statistics 2018: GLOBOCAN estimates of incidence and mortality worldwide for 36 cancers in 185 countries. CA: a cancer journal for clinicians 2018, 68(6):394-424.

3. Siegel RL, Miller KD, Jemal A: Cancer statistics, 2020. CA: a cancer journal for clinicians 2020, 70(1):7-30.

4. Tam S, Boonsripitayanon M, Amit M, Fellman BM, Li Y, Busaidy NL, Cabanillas ME, Dadu R, Sherman S, Waguespack SG et al: Survival in Differentiated Thyroid Cancer: Comparing the AJCC Cancer Staging Seventh and Eighth Editions. Thyroid : official journal of the American Thyroid Association 2018, 28(10):1301-1310. 
5. See A, lyer NG, Tan NC, Teo C, Ng J, Soo KC, Tan HK: Distant metastasis as the sole initial manifestation of well-differentiated thyroid carcinoma. European archives of oto-rhino-laryngology : official journal of the European Federation of Oto-Rhino-Laryngological Societies (EUFOS) : affiliated with the German Society for Oto-Rhino-Laryngology - Head and Neck Surgery 2017, 274(7):28772882.

6. Qutbi M, Shafeie B, Amoui M, Tabeie F, Azizmohammadi Z, Mahmoud-Pashazadeh A, Javadi $H$, Assadi M, Asli IN: Evaluation of Prognostic Factors Associated With Differentiated Thyroid Carcinoma With Pulmonary Metastasis. Clinical nuclear medicine 2016, 41(12):917-921.

7. Orita Y, Sugitani I, Toda K, Manabe J, Fujimoto Y: Zoledronic acid in the treatment of bone metastases from differentiated thyroid carcinoma. Thyroid : official journal of the American Thyroid Association 2011, 21(1):31-35.

8. Osorio M, Moubayed SP, Su H, Urken ML: Systematic review of site distribution of bone metastases in differentiated thyroid cancer. Head \& neck 2017, 39(4):812-818.

9. Orita Y, Sugitani I, Matsuura M, Ushijima M, Tsukahara K, Fujimoto Y, Kawabata K: Prognostic factors and the therapeutic strategy for patients with bone metastasis from differentiated thyroid carcinoma. Surgery 2010, 147(3):424-431.

10. Lee J, Soh EY: Differentiated thyroid carcinoma presenting with distant metastasis at initial diagnosis clinical outcomes and prognostic factors. Annals of surgery 2010, 251(1):114-119.

11. Durante C, Haddy N, Baudin E, Leboulleux S, Hartl D, Travagli JP, Caillou B, Ricard M, Lumbroso JD, De Vathaire $\mathrm{F}$ et al: Long-term outcome of $\mathbf{4 4 4}$ patients with distant metastases from papillary and follicular thyroid carcinoma: benefits and limits of radioiodine therapy. The Journal of clinical endocrinology and metabolism 2006, 91(8):2892-2899.

12. Song HJ, Wu CG, Xue YL, Xu YH, Qiu ZL, Luo QY: Percutaneous osteoplasty combined with radioiodine therapy as a treatment for bone metastasis developing after differentiated thyroid carcinoma. Clinical nuclear medicine 2012, 37(6):e129-133.

13. Do MY, Rhee Y, Kim DJ, Kim CS, Nam KH, Ahn CW, Cha BS, Kim KR, Lee HC, Park CS et al: Clinical features of bone metastases resulting from thyroid cancer: a review of 28 patients over a 20-year period. Endocrine journal 2005, 52(6):701-707.

14. Zettinig G, Fueger BJ, Passler C, Kaserer K, Pirich C, Dudczak R, Niederle B: Long-term follow-up of patients with bone metastases from differentiated thyroid carcinoma - surgery or conventional therapy? Clin Endocrinol 2002, 56(3):377-382.

15. Han E, Lee YY, Kim E, Kwon SY, Kim SP, Kim HS, Kim MK: Solitary bone and brain metastasis in a patient with papillary thyroid carcinoma mimicking cavernous angioma. The Korean journal of internal medicine 2016, 31(5):991-994.

16. Varadarajan VV, Pace EK, Patel V, Sawhney R, Amdur RJ, Dziegielewski PT: Follicular thyroid carcinoma metastasis to the facial skeleton: a systematic review. BMC cancer 2017, 17(1):225.

17. Zhang WD, Liu DR, Feng CC, Zhou CB, Zhan CN, Que RS, Chen L: Management of differentiated thyroid carcinoma with bone metastasis: a case report and review of the Chinese literature. Journal 
of Zhejiang University Science B 2014, 15(12):1081-1087.

18. Surveillance, Epidemiology, and End Results (SEER) Program (www.seer.cancer.gov) SEER*Stat Database: Incidence - SEER 18 Regs Custom Data (with additional treatment fields), Nov 2018 Sub (1975-2016 varying) - Linked To County Attributes - Total U.S., 1969-2017 Counties, National Cancer Institute, DCCPS, Surveillance Research Program, released April 2019, based on the November 2018 submission.

19. Zheng X-Q, Huang J-F, Lin J-L, Chen L, Zhou T-T, Chen D, Lin D-D, Shen J-F, Wu A-M: Incidence, prognostic factors, and a nomogram of lung cancer with bone metastasis at initial diagnosis: a population-based study. Transl Lung Cancer Res 2019, 8(4):367-379.

20. Kennecke H, Yerushalmi R, Woods R, Cheang MCU, Voduc D, Speers CH, Nielsen TO, Gelmon K: Metastatic behavior of breast cancer subtypes. J Clin Oncol 2010, 28(20):3271-3277.

21. Liede A, Jerzak KJ, Hernandez RK, Wade SW, Sun P, Narod SA: The incidence of bone metastasis after early-stage breast cancer in Canada. Breast Cancer Res Treat 2016, 156(3):587-595.

22. Tickoo SK, Pittas AG, Adler M, Fazzari M, Larson SM, Robbins RJ, Rosai J: Bone metastases from thyroid carcinoma: a histopathologic study with clinical correlates. Arch Pathol Lab Med 2000, 124(10):1440-1447.

23. Lang BH, Wong KP, Cheung CY, Wan KY, Lo CY: Evaluating the prognostic factors associated with cancer-specific survival of differentiated thyroid carcinoma presenting with distant metastasis. Annals of surgical oncology 2013, 20(4):1329-1335.

24. Slook O, Levy S, Slutzky-Shraga I, Tsvetov G, Robenshtok E, Shimon I, Benbassat C, Hirsch D: LONGTERM OUTCOMES AND PROGNOSTIC FACTORS IN PATIENTS WITH DIFFERENTIATED THYROID CARCINOMA AND BONE METASTASES. Endocrine practice : official journal of the American College of Endocrinology and the American Association of Clinical Endocrinologists 2019, 25(5):427-437.

25. Choi YM, Kim WG, Kwon H, Jeon MJ, Lee JJ, Ryu JS, Hong EG, Kim TY, Shong YK, Kim WB: Early prognostic factors at the time of diagnosis of bone metastasis in patients with bone metastases of differentiated thyroid carcinoma. European journal of endocrinology 2016, 175(3):165-172.

26. Bernier MO, Leenhardt L, Hoang C, Aurengo A, Mary JY, Menegaux F, Enkaoua E, Turpin G, Chiras J, Saillant $\mathrm{G}$ et al: Survival and therapeutic modalities in patients with bone metastases of differentiated thyroid carcinomas. The Journal of clinical endocrinology and metabolism 2001, 86(4):1568-1573.

27. Vuong HG, Duong UNP, Pham TQ, Tran HM, Oishi N, Mochizuki K, Nakazawa T, Hassell L, Katoh R, Kondo T: Clinicopathological Risk Factors for Distant Metastasis in Differentiated Thyroid Carcinoma: A Meta-analysis. World journal of surgery 2018, 42(4):1005-1017.

28. Dralle H, Machens A, Basa J, Fatourechi V, Franceschi S, Hay ID, Nikiforov YE, Pacini F, Pasieka JL, Sherman SI: Follicular cell-derived thyroid cancer. Nature reviews Disease primers 2015, 1:15077.

29. Goffredo P, Sosa JA, Roman SA: Differentiated thyroid cancer presenting with distant metastases: a population analysis over two decades. World journal of surgery 2013, 37(7):1599-1605. 
30. Nakayama R, Horiuchi K, Susa M, Watanabe I, Watanabe K, Tsuji T, Matsumoto M, Toyama Y, Morioka $\mathrm{H}$ : Clinical outcome after bone metastasis (BM) surgery in patients with differentiated thyroid carcinoma (DTC): a retrospective study of $\mathbf{4 0}$ cases. Japanese journal of clinical oncology 2014, 44(10):918-925.

31. Nixon IJ, Ganly I, Shah JP: Thyroid cancer: surgery for the primary tumor. Oral oncology 2013, 49(7):654-658.

32. Kluijfhout WP, Rotstein LE, Pasternak JD: Well-differentiated thyroid cancer: Thyroidectomy or lobectomy? CMAJ : Canadian Medical Association journal = journal de l'Association medicale canadienne 2016, 188(17-18):E517-e520.

33. Schmidt M, Gorges R, Drzezga A, Dietlein M: A Matter of Controversy: Is Radioiodine Therapy Favorable in Differentiated Thyroid Carcinoma? J Nucl Med 2018, 59(8):1195-1201.

34. Podnos YD, Smith D, Wagman LD, Ellenhorn JD: Radioactive iodine offers survival improvement in patients with follicular carcinoma of the thyroid. Surgery 2005, 138(6):1072-1076; discussion 10761077.

35. Ilhan $H$, Mustafa $M$, Bartenstein $P$, Kuwert $T$, Schmidt $D$ : Rate of elimination of radioiodine-avid lymph node metastases of differentiated thyroid carcinoma by postsurgical radioiodine ablation. A bicentric study. Nuklearmedizin Nuclear medicine 2016, 55(6):221-227.

36. Qiu ZL, Song HJ, Xu YH, Luo QY: Efficacy and survival analysis of 131/ therapy for bone metastases from differentiated thyroid cancer. The Journal of clinical endocrinology and metabolism 2011, 96(10):3078-3086.

37. Ma C, Feng F, Wang S, Fu H, Wu S, Ye Z, Chen S, Wang H: Chinese Data of Efficacy of Low- and HighDose lodine-131 for the Ablation of Thyroid Remnant. Thyroid : official journal of the American Thyroid Association 2017, 27(6):832-837.

38. Kondraciuk JD, Rice SL, Zhou X, Gharzeddine K, Knezevic A, Spratt DE, Sabra M, Larson SM, Grewal RK, Osborne JR: Thyroid Cancer Bone Metastasis: Survival and Genomic Characteristics of a Large Tertiary Care Cohort. Clinical nuclear medicine 2019, 44(8):e465-e471.

39. Ibrahim EY, Busaidy NL: Treatment and surveillance of advanced, metastatic iodine-resistant differentiated thyroid cancer. Current opinion in oncology 2017, 29(2):151-158.

40. Mikalsen LTG, Arnesen MR, Bogsrud TV, Dale E, Stokke C: Combining radioiodine and external beam radiation therapy: the potential of integrated treatment planning for differentiated thyroid cancer. Acta oncologica (Stockholm, Sweden) 2017, 56(6):894-897.

\section{Figures}


SEER*Stat Database: Incidence - SEER 18 Regs Custom Data (2010-2016 varying)

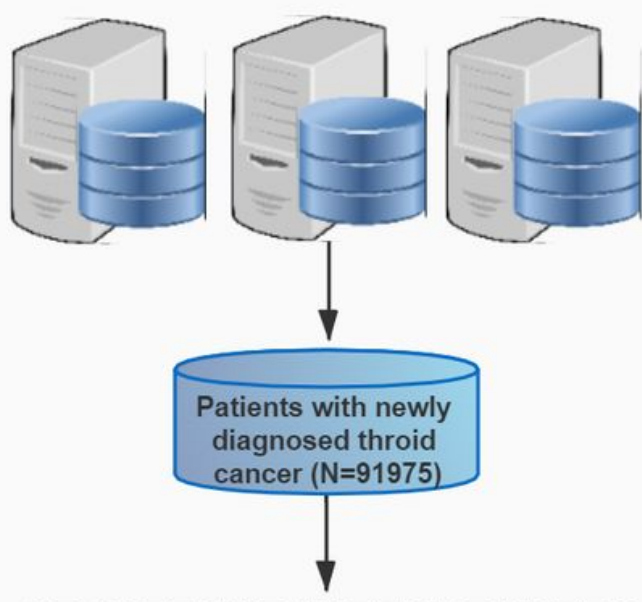

Excluded

1. Age $<18$ years old $(\mathrm{N}=1044)$;

2. Not the primary cancer $(\mathrm{N}=12427)$;

1 3. Diagnosis without positive histology

I confirmation $(\mathrm{N}=2697)$;

1 4. Without specific information of TNM

I system $(\mathrm{N}=4860)$;

5. Diagnosis without ICD-O-3 histology

code of DTC $(\mathrm{N}=3552)$;

6. Diagnosis with unknown bone and lung metastases $(\mathrm{N}=219)$;
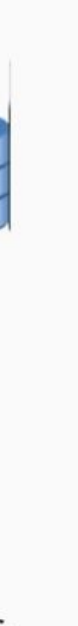

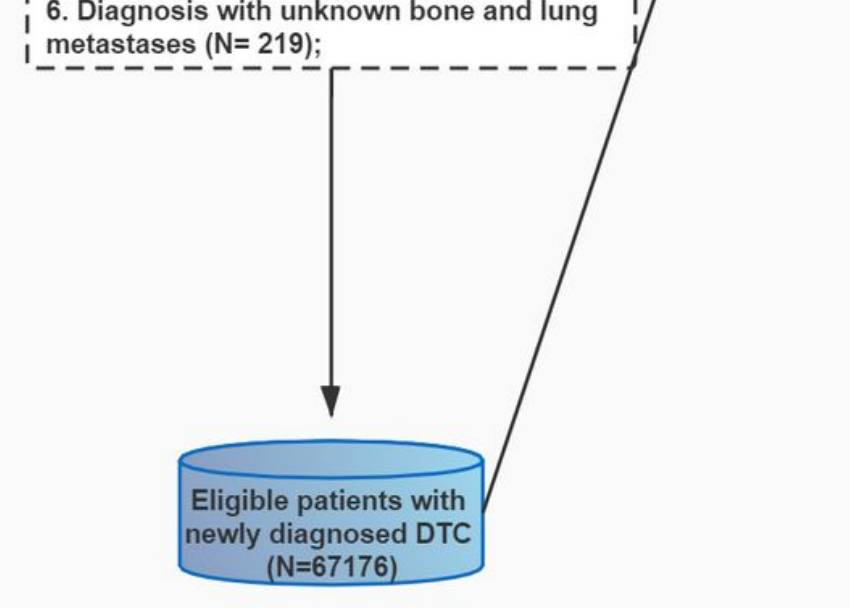

Patients with DTC diagnosed with BM. $(\mathrm{N}=244)$

Patients with DTC diagnosed without $\mathrm{BM}$. $(\mathrm{N}=66932)$

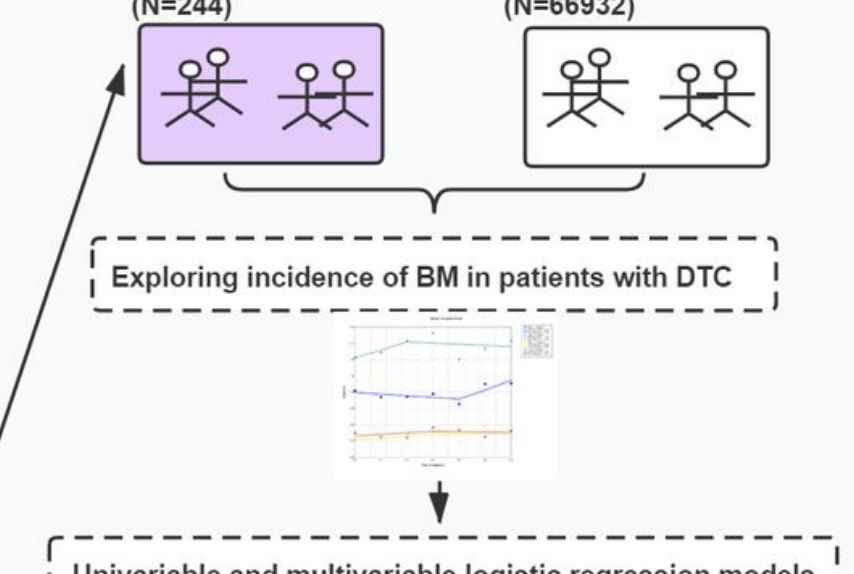

Univariable and multivariable logistic regression models - - - - - - - - - - T-- - - - - - - - -
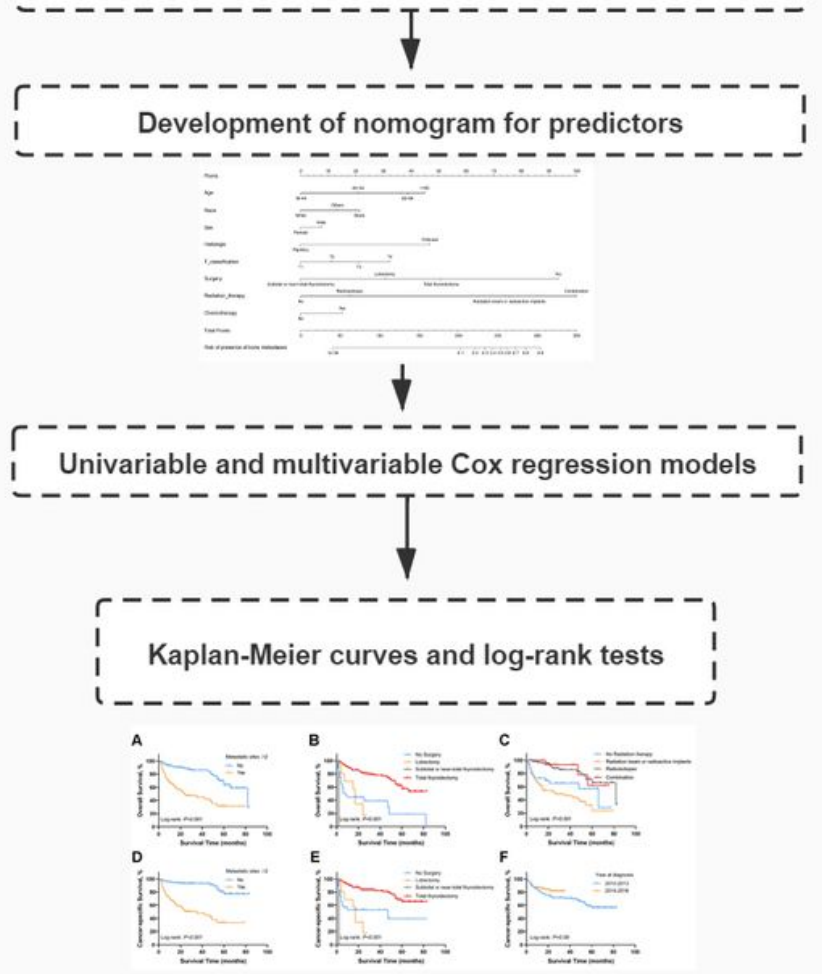

\section{Figure 1}

Study design 
Multiple Joinpoint Models

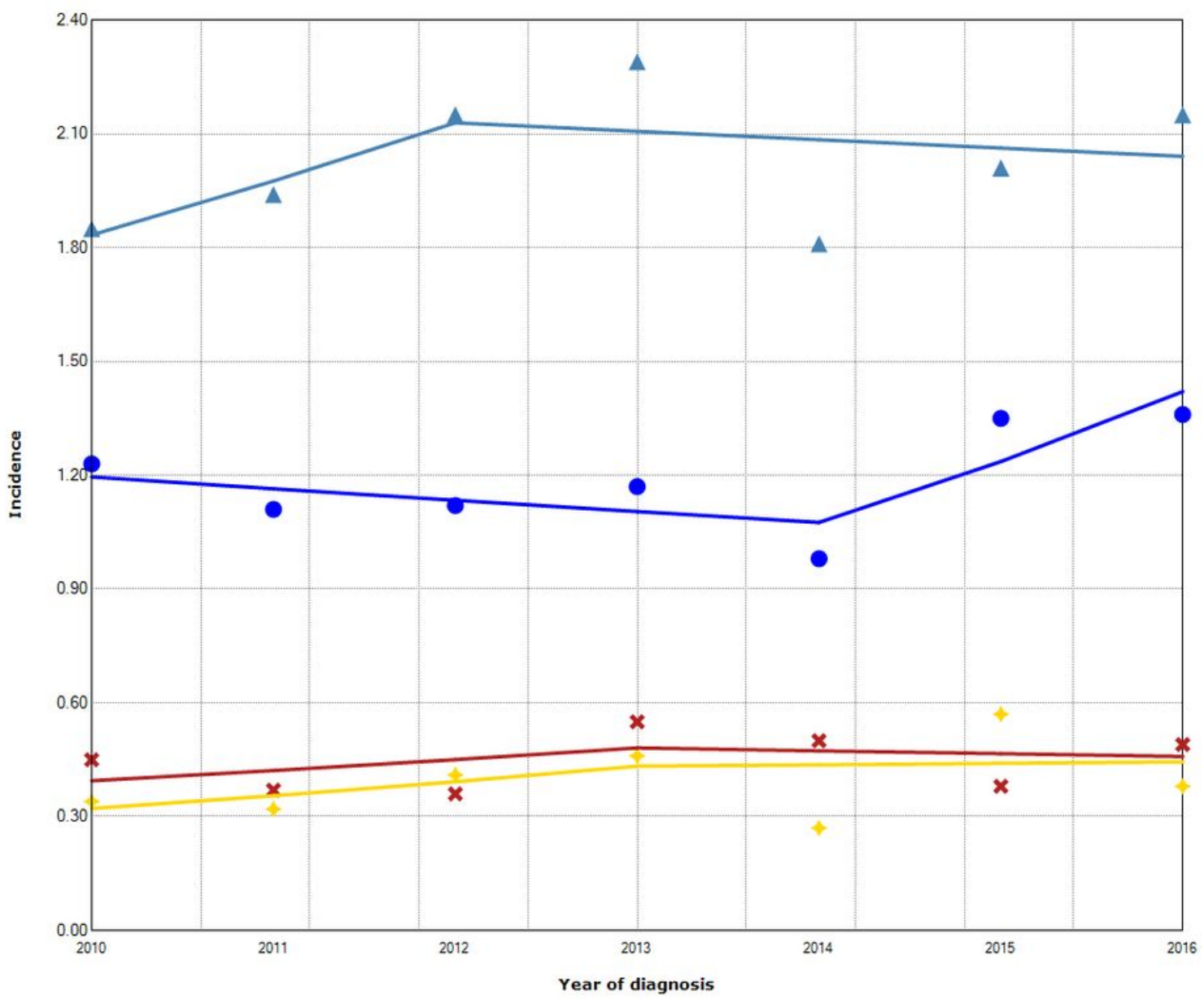

- Bone - 1 Joinpoint

2010-2014 $\mathrm{APC}=-2.61$

4 lung-1 Joinpoint

2010-2012 APC $=7.76$

$2012-2016$ APC $=-1.05$

2010.2013 APC $=10.43$

2013.2016 APC $=0.86$

Liver - 1 Joinpoint

2010-2013 APC $=6.83$

- 2013-2016 APC $=-1.57$

\section{Figure 2}

Trends in annual incidence rates of DTC with different distant metastasis to specific sites 
Points

Age

Race

Sex

Histologic

T_classification

Surgery

Radiation_therapy

Chemotherapy

Total Points

Risk of presence of bone metastases
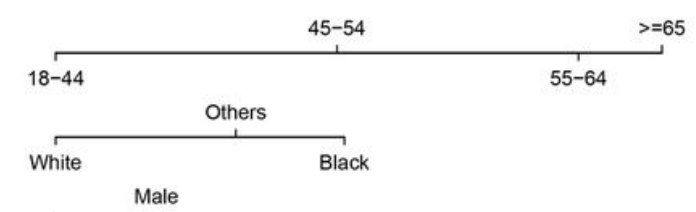

Female

Papillary
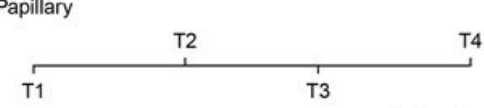

Subtotal or near-total thyroidectomy

Lobectomy

remy

Total thyroidectomy

Radioisotopes

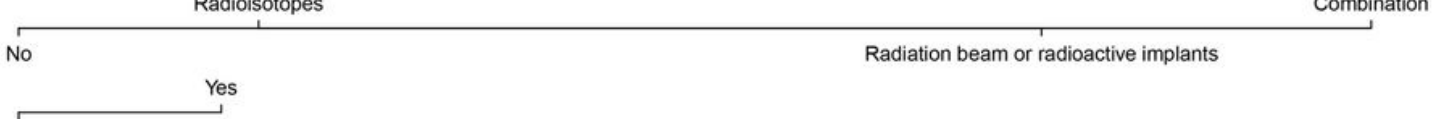

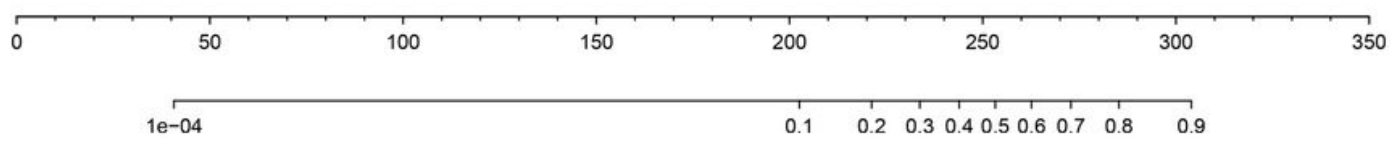

Figure 3

Nomogram for predictors of presence of BM in the patients with DTC

A

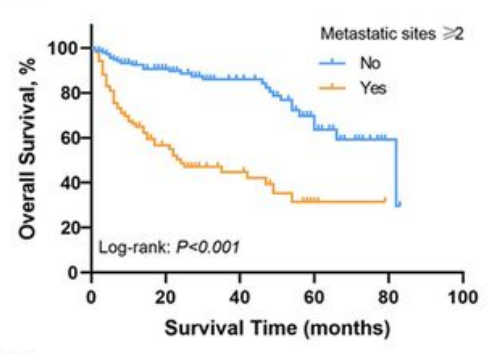

D

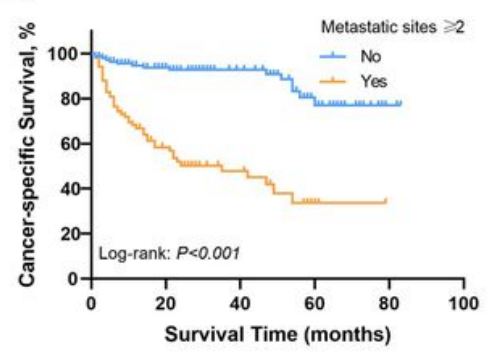

B

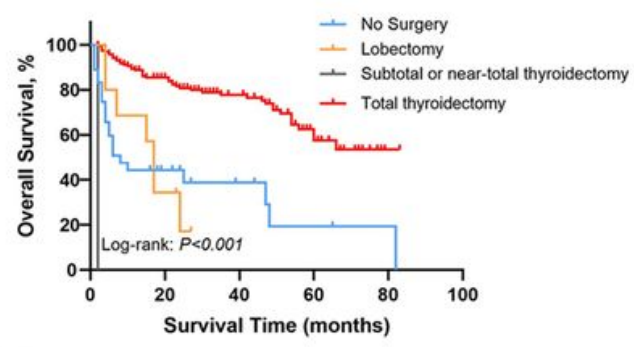

E

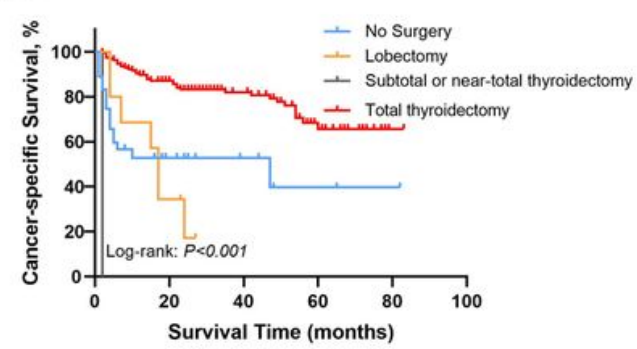

C

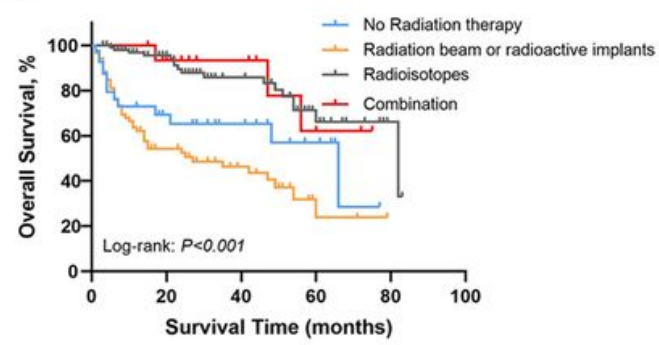

$\mathbf{F}$

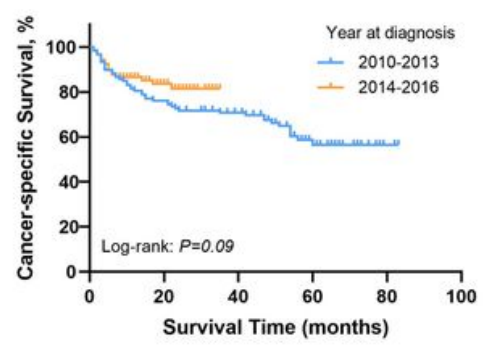

\section{Figure 4}

Kaplan-Meier survival curves presenting OS and CSS of in Patients with DTC diagnosed with BM. (A) OS between metastasis sites $\geq 2$ and $\mathbb{2} 2$. (B) OS in different surgery approaches. (C) OS in different radiation

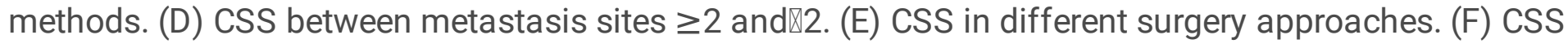
in different radiation methods. 


\section{Supplementary Files}

This is a list of supplementary files associated with this preprint. Click to download.

- supplementarymaterials.docx 